Accepted refereed manuscript of:

Meginnis K \& Campbell D (2017) Students' preferences for attributes of postgraduate economics modules: Evidence from a multi-profile best-worst scaling survey, International Review of Economics Education, 24, pp. 18-27.

DOI: $10.1016 /$ j.iree.2016.11.001

(C) 2016, Elsevier. Licensed under the Creative Commons AttributionNonCommercial-NoDerivatives 4.0 International http://creativecommons.org/licenses/by-nc-nd/4.0/ 


\title{
Students' preferences for attributes of postgraduate economics modules: evidence from a multi-profile best-worst scaling survey
}

\author{
Keila Meginnis ${ }^{1}$ \\ Environmental and Resource Economics Research Group, School of Social Sciences, University of \\ Manchester, UK \\ Danny Campbell ${ }^{2}$
}

Economics Division, Stirling Management School, University of Stirling, UK

Paper accepted in International Review of Economics Education, $2^{\text {st }}$ November 2016

\begin{abstract}
In this study, we investigate Scottish postgraduate economics students' preferences for module design. Using a multi-profile best-worst scaling survey, we find that students have clear preferences on how they wish their modules to be delivered, taught and assessed. Furthermore, using a discrete mixtures modelling approach we explain the heterogeneous nature of preferences for the module attributes and the students' lexicographic preference orderings. We show how failing to address this leads to erroneous results and limits the ability to derive reliable prediction. The findings in this study should appeal to university staff involved in the design of postgraduate (as well as undergraduate) courses as it should help them better establish a coherent learning experience for students, through which students can attain their full academic potential.
\end{abstract}

JEL classification: C25, I21, I23

Keywords: Taught postgraduate; module choice; student's preferences; multi-profile best-worst scaling; discrete mixtures model; attribute non-attendance

Highlights

- We use multi-profile best-worst scaling survey to investigate students' preferences

- We find student preferences depend on how modules are delivered, taught and assessed

- There is heterogeneity in students preferences and the decision heuristics they adopt

\section{Introduction}

Since the 1990s, postgraduate education has grown in popularity due, in part, to the increasing wage gap between high school graduates and university graduates and subsequently, between university graduates and postgraduates (Donaldson and McNicholas, 2004; Lemieux, 2006; Ho et al., 2012; Morgan, 2014). In addition, the curriculum of undergraduate courses has broadened, due to increased pressure on institutions to provide graduates with a wider range of abilities, viewpoints and transferable skills. This has consequentially led to an increase in the number of students seeking to undertake postgraduate degrees. These degrees provide more specialised knowledge suitable for their professional career, which is increasingly lacking in undergraduate degrees programmes (Ho et al., 2012; Kember et al., 2016). While postgraduate degrees generally enhance student employability, the fees, as well as the potential opportunity costs, can be substantial. Postgraduate degrees are usually self-financed through loans, employment and parental contributions. Moreover, many of the students enrolling in postgraduate degrees come from abroad and, thus, typically pay considerably

\footnotetext{
${ }^{1}$ Author for correspondence: Oxford Road, Manchester M13 9PL, UK; tel: +44(0)7925813797; email: keila.meginnis@postgrad.manchester.ac.uk

${ }^{2}$ Stirling, FK9 4LA, UK; email: danny.campbell@ stir.ac.uk
} 
higher fees (in fact, these can be more than double the domestic fee rate (Kember et al., 2016)). Therefore, it is essential that postgraduate degrees, which likely attract dedicated and hard-working students, offer value for money and that all the expectations of students pursuing taught postgraduate degrees are met.

Meeting the growing demand for postgraduate education is a key priority for many universities. However, to do so requires that they have a thorough understanding of students' preferences surrounding different aspects of postgraduate education. If students have strong preferences for one type of education, then universities would benefit by tailoring their courses to attract more postgraduate students. Additionally, universities strive to rank highly across several high profile national surveys that collect data on student experiences and satisfaction (e.g., the National Student Survey (see Canning, 2015; Douglas et al., 2015, for futher details) and the Postgraduate Taught Experience Survey (see Leman et al., 2013) are conducted annually across the UK). Postgraduate modules that are designed according to students' preferences are likely to get higher student satisfaction marks, which should, ultimately, help universities achieve higher rankings from their students. This motivates our work. In this paper, we aim to determine students' preferences for how their postgraduate modules are delivered, taught and assessed. We acknowledge that while course design is also contingent on administrative factors (such as room availability, instructor availability etc.), we argue that students exhibit preferences for factors which course conveners can easily adjust according to preferences. While course design must also be structured such that it meets the course objectives, accounting for students preferences when possible will ultimately facilitate learning and increase student satisfaction.

A number of previous studies have primarily focused on identifying the attributes that influence university attendance (e.g., Holdsworth and Nind, 2006; Hagel and Shaw, 2010; Dunnett et al., 2012). However, there is limited evidence on students' preferences for the different attributes of the courses or modules offered at universities. Therefore, our study is a novel application and makes an important contribution to the literature on the delivery of higher educational courses. Not unlike Flannery et al. (2013), we use a stated preference elicitation method to establish preferences for a range of postgraduate course characteristics. Specifically, this involves describing postgraduate modules using a number of attributes, each of which is denoted using various levels. We focus on attributes relating to how the module is delivered, taught and assessed. However, our approach differs from that used by Flannery et al. (2013) in the sense that we use a multi-profile best-worst scaling approach. This has the important advantage of providing additional information about attribute levels that make a module unacceptable. To the best of our knowledge, this is the first application of this approach in the education literature.

A fundamental assumption behind this choice framework is that people consider all attributes, make tradeoffs between them and choose the alternative that offers them the best combination of attributes. However, recent studies (e.g., Hensher et al., 2005; Campbell et al., 2008; Scarpa et al., 2009; Campbell et al., 2011; Hensher et al., 2012b; Hess and Hensher, 2013; Scarpa et al., 2013) have recognised that when people make choices, there is a tendency for some of them to consider only a subset of the attributes. Some of the underlying reasons, which have been explored in Alemu et al. (2013) include, inter alia, (i) a genuine disinterest in the attributes, (ii) the context and survey design related issues, such as complexity, controversy and sensitivity of the survey topic, irrelevance of the attribute and the cognitive demand required to complete choice tasks, (iii) peoples' different capabilities and motivations (Hensher et al., 2005), and (iv) strategic behaviour people may exhibit. Notwithstanding the recent extension of the analysis of this type of behaviour in a range of disciplines, the topic has yet to be considered when studying students' preferences. Nevertheless, attribute non-attendance in module and course choice is potentially highly relevant. Indeed, it would not be surprising for students to use lexicographic preference orderings or other decision-making heuristics when deciding which module to enrol in. In this paper, we accommodate this type of behaviour and identify the proportion of students who adopt it.

Our sample includes students enrolled in taught postgraduate economics courses in Scotland. Our findings correspond with those established by Kember et al. (2016), who found a disconnect between students' expectations and their actual learning experience. We observe that, on average, students have strongest preferences for modules that are not delivered in lengthy blocks and that they dislike modules without tutorials and those that have a small component of coursework assessment. Nevertheless, we find significant heterogeneity in preferences among students and a significant share who ignore the module attributes when they made their module choices. 
The remainder of this paper is organised as follows. In Section 2, we outline our empirical case-study. In Section 3 we describe our approach to this issue. Section 4 reports estimation and post-estimation results, while Section 5 discusses the implications of these findings and concludes.

\section{Survey design}

Similar to Flannery et al. (2013) we use a stated preference elicitation method. While Flannery et al. (2013) implement a discrete choice experiment, we implement a multi-profile best-worst scaling approach. This method has been used, and shown to be suitable, in a number of research areas to assess people's perception of intangible concepts. In this paper, we use the method to investigate students' preferences for postgraduate module attributes. To the best of our knowledge, this is the first application of multi-profile best-worst scaling for exploring module choice.

In multi-profile best-worst scaling, developed by Finn and Louviere (1992), respondents are asked to choose their most and least preferred alternatives among several hypothetical alternatives in a choice set, and are typically asked to perform a sequence of such choices. Experimental design theory is used to construct the alternatives, which are defined in terms of their attributes and the levels these attributes can take. The method can, thus, be used to examine the response of a student to changes in each of the module attributes. In other words, it is possible to infer their willingness to give up some amount of one attribute in order to achieve more of another (i.e., their marginal rate of substitution between the attributes). The theoretical foundation of multi-profile best-worst scaling combines several different theories (see Louviere et al., 2015, for a thorough explanation). They are consistent with the Lancaster's (1966) microeconomic approach, whereby individuals derive utility from the different characteristics, or attributes, that a good possesses, rather than directly from the good per se. Accordingly, a change in one of the attributes can cause a student to switch their decision from one module to another that provides a superior combination of attributes. Based on the characteristics theory of value proposed by Lancaster, the probability of choosing a specific module is a function of the utility associated with that module. Moreover, it is assumed that the utility derived from each module is determined by the preferences over the levels of the characteristics provided by that module. The choice model is usually derived under the assumption that individuals behave in a utility-maximising manner. The origins of probabilistic choice goes back to the work of Thurstone (1927), who developed the concepts in terms of psychological stimuli. Thurstone proposed the modelling of individual choice as the outcome of a process in which a random variable is associated with each alternative, and the alternative with the highest realisation is the one selected. When the perceived stimuli are interpreted as levels of satisfaction, or utility, this can be interpreted as a model for economic choice in which the individual chooses the alternative yielding the greatest realisation of utility (McFadden, 2001). Thurstone's work was introduced into economics by exploring the theoretical implications of choice probabilities for the maximisation of utilities that contained random elements (named random utility models). This idea was later taken up and further developed by McFadden (1974) and Manski (1977).

In this study, postgraduate students completed an online multi-profile best-worst scaling survey regarding module preferences. The experiment consisted of three attributes with three levels each. The attributes and corresponding levels were chosen after discussions with current postgraduate students regarding their modules. The following three attributes were selected as relevant determinants of a postgraduate module: (i) delivery; (ii) teaching; and, (iii) assessment. ${ }^{3}$

The survey offered descriptions of all attributes. Delivery was defined as how often the course meets over a semester and for how many hours. The three levels of delivery were: forty 1-hour sessions; twenty 2-hour sessions; and ten 4-hour sessions. The second attribute, teaching, was how the course is divided between lectures, tutorials, or lab sessions. The three levels were: lectures, tutorials, and lab sessions; lectures and lab sessions; and lectures and tutorials. ${ }^{4}$

\footnotetext{
${ }^{3}$ We deliberately chose not to include a price attribute because the principal aim of this study was to explore preferences among students who had are already enrolled at university. Had the study been designed to identify preferences for different universities, where tuition fees vary, the inclusion of a price attribute would have been appropriate. This said, we note that a number of studies find the tuition fees to be an insignificant attribute (Holdsworth and Nind, 2006; Dunnett et al., 2012).

${ }^{4}$ In Scotland, lectures are generally delivered by the professor and principally focus on the critical information, background, and theories; tutorials are usually given by $\mathrm{Ph}$.D. students who review homework assignments and include interactive problem solving
} 
Consider the three modules shown below

Holding all else constant, please indicate which of the three you MOST and LEAST prefer

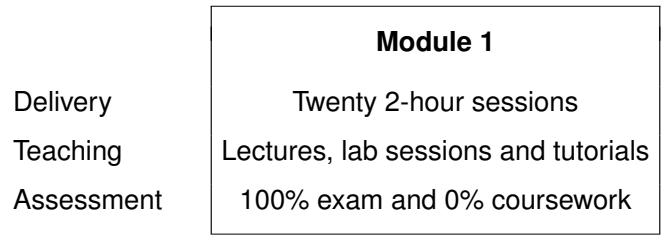

Most preferred

Least preferred

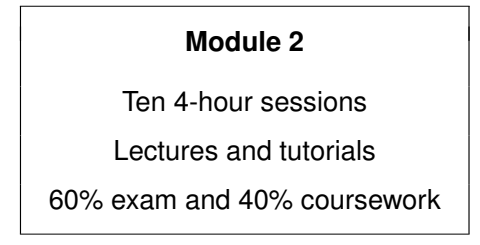

$\mathrm{O}$

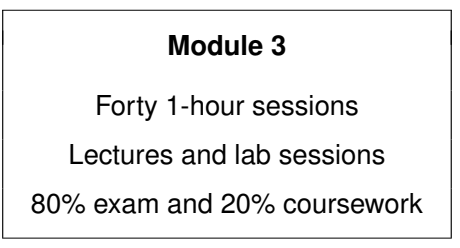

$\bigcirc$

Figure 1: Typical best-best choice task used in the study

As noted by Donaldson and McNicholas (2004), the method of assessment is a significant factor of students choice of postgraduate degrees. For this reason, the final attribute corresponded to how the course is assessed, either through examinations and/or coursework (e.g. homework or group assignments). As the study focused on postgraduate attributes, we chose assessment levels that are most typically used in a postgraduate economics module. The levels of assessment were: 100 percent examination and 0 percent coursework; 80 percent examination and 20 percent coursework; and 60 percent examination and 40 percent coursework.

An online survey was designed and administered to postgraduate students taking economics modules in Scottish universities. The survey was advertised through social media and course email lists. Each student was presented with eight choice tasks. Each choice task showed three different economics modules that differed between levels of delivery, assessment, and teaching. Students were asked to choose their most and least preferred module out of the three choices given. An example of a multi-profile best-worst scaling used in our study is shown in Figure 1.

Fifty-nine postgraduate students completed the survey meaning that the final number of best and worst choice observations for analysis is 944 . In addition to the multi-profile best-worst scaling tasks, students answered a number of socio-economic questions relating to their nationality, income and education experience, summary results of which are reported in Table 1 . The sample consists of 48 percent female and 52 percent male students with a mean age of 24.8 years, which is in line with a United Nations Census of students enrolled in postgraduate studies in the UK (Morgan, 2014). Only 10 percent of the sampled students have part-time work. We asked students whether they paid international or domestic fees and found that 42 percent paid international fees. ${ }^{5}$

\section{Econometric approach}

Multi-profile best-worst scaling is an application of the random utility maximisation theory (Manski, 1977; Thurstone, 1927), whereby students are assumed to evaluate all possible pairs of profiles within the displayed choice task and choose the pair that reflects their maximum difference in preference. The number of unique pairs, $J$, is given by $S(S-1)$, where $S$ represents the number of modules in the choice task. Overall utility, $U$, which is defined by $K$ attributes, $x_{k}$, and where $k=\{1,2,3, \ldots, K\}$, is associated with student $n$ 's chosen pair, $i$, in task $t$ is given by the difference in utility between the best and worst profiles:

$$
U_{n i t}=\underbrace{\left(\sum_{k=1}^{K} \sum_{l=1}^{L} \beta_{k_{l}} x_{b_{n i k_{l}}}\right)}_{\text {Best }}-\underbrace{\left(\sum_{k=1}^{K} \sum_{l=1}^{L} \beta_{k_{l}} x_{w_{n i k_{l}}}\right)}_{\text {Worst }}+\varepsilon_{\text {nit }},
$$

where $\beta_{k_{l}}$ is an estimated parameter associated with the dummy-coded $l^{\text {th }}$ level of attribute $k, x_{k_{l}}$ (indexed by $b$ or $w$ to distinguish between best and worst profiles respectively), and $\varepsilon$ is an iid type I extreme value (EV1)

sessions; and, lab sessions are given by either the professor or tutor and apply material from the lectures to real work applications (e.g., with the aid of statistical programs and/or other relevant resources).

${ }^{5}$ In the UK, international fees are paid by non-EU students. 


\section{Table 1}

Summary of sampled student's characteristics

Number (Share)

\section{Gender}

Female

Male

Age

$\leq 23$ years of age

24 years of age

25 years of age

$\geq 26$ years of age

\section{Part-time work}

No

Yes

Tuition fees

Domestic

International

$25(0.42)$

distributed error term, with constant variance of $\pi^{2} / 6$. As our attributes are qualitative, the values of $\beta_{k_{l}}$ are subject to the constraint $\sum_{l=1}^{L} \beta_{k_{l}}=0$ for identification purposes, which is equivalent to effects coding (i.e., to avoid perfect multicollinearity, we arbitrarily express one of the attribute levels as the negative sum of the other levels).

Given these assumptions, the probability of the sequence of best-worst choices made by individual $n$ can be represented by the multinomial logit model:

$$
\operatorname{Pr}\left(y_{n} \mid x_{n}\right)=\prod_{t=1}^{T_{n}} \frac{\exp \left[\left(\sum_{k=1}^{K} \sum_{l=1}^{L} \beta_{k_{l}} x_{b_{n i t k_{l}}}\right)-\left(\sum_{k=1}^{K} \sum_{l=1}^{L} \beta_{k_{l}} x_{w_{n i t k_{l}}}\right)\right]}{\sum_{j=1}^{J} \exp \left[\left(\sum_{k=1}^{K} \sum_{l=1}^{L} \beta_{k_{l}} x_{b_{n j t k_{l}}}\right)-\left(\sum_{k=1}^{K} \sum_{l=1}^{L} \beta_{k_{l}} x_{w_{n j k_{l}}}\right)\right]},
$$

where $y_{n}$ gives the sequence of best-worst choices over the $T_{n}$ choice tasks for student $n$, i.e., $y_{n}=$ $\left\langle i_{n 1}, i_{n 2}, \ldots, i_{n T_{n}}\right\rangle .^{6}$

The choice probability retrieved from Eq. 2 assumes all students share the same preferences for the module attributes. In this paper, we are interested in explaining the heterogeneous nature of preferences for the module attributes among the sample of students as well as possible attribute non-attendance. Such (unobserved) preference heterogeneity can be accommodated by assuming random distributions. Rather than continuous random distributions, we opt for finite (discrete) distributions. The advantage of this nonparametric approach is that commonly used continuous distributions may be unsuitable for representing the distribution of preferences, especially in situations where there are spikes in the distribution. Finite distributions-instead-can provide greater flexibility and have practical appeal as the results can have more intuitive meaning than the parameters and moments of the distributions retrieved from continuous parametric distributions. We acknowledge the similarity between the discrete mixtures model and the latent class logit model, which also assume finite representations of heterogeneity. In fact, both models are formally equivalent, the main difference being that in discrete mixtures models the focus is usually on segmenting on a per parameter basis and not on the basis of the full set of parameters, which is typically the case in latent class models. Indeed, both specifications can be estimated using a number of equality constraints. We favour the behavioural appeal of retrieving probabilistic estimates for each parameter directly afforded by the discrete mixtures approach.

\footnotetext{
${ }^{6}$ We note that accounting for the panel effect is immaterial in the multinomial logit model due to the independence of choice probabilities. We, nevertheless, present the multinomial logit model in this manner to introduce the necessary terms as early on as possible so that differences in models are clearer as we progress through this section.
} 
In a discrete mixtures context, the number of possible values for the parameter coefficients is finite (Hess et al., 2007). Therefore, discrete mixtures specifications are especially suited for identifying and accommodating segments of students based on their underlying preferences. This can be accommodated by specifying the vector of parameters relating to attribute $k, \beta_{k}$, as having $M_{k}$ mass points, $\beta_{k}^{m}$, where $m=\left\{1,2,3, \ldots, M_{k}\right\}$. A student's true preferences cannot be known with certainty and, thus, remains latent. To work around this, based on observed choice behaviour, the presence of each vector of parameters can be established up to a probability, with the full probability per student allocated across all $M_{k}$ classes. The unconditional probability of observing $\beta_{k}^{m}$ is denoted by $\pi_{k}^{m}$, subject to $\sum_{m=1}^{M_{k}} \pi_{k}^{m}=1$ (i.e., the prior likelihood of competing marginal utilities being their actual marginal utilities). ${ }^{7}$

The number of possible segments with $K$ attributes is $Q=\prod_{k=1}^{K} M_{k}$. Each segment, $q=\{1,2,3, \ldots, Q\}$, implies a different combination of attribute marginal utilities for each of the $K$ attributes. The unconditional probability of observing combination $q$ is the product of the unconditional probabilities of observing each $\pi_{k}^{m}$ and is denoted using $\phi_{q}$. For example, the probability of observing $\beta_{1}^{3}, \beta_{2}^{1}$ and $\beta_{3}^{2}$ is given by $\phi_{q}=\pi_{1}^{3} \times \pi_{2}^{1} \times \pi_{3}^{2}$. The probability of a sequence of choices can then be rewritten as:

$$
\operatorname{Pr}\left(y_{n} \mid x_{n}\right)=\sum_{q=1}^{Q} \phi_{q} \prod_{t=1}^{T_{n}} \frac{\exp \left[\left(\sum_{k=1}^{K} \sum_{l=1}^{L} \beta_{k_{l}}^{q} x_{b_{n i t k_{l}}}\right)-\left(\sum_{k=1}^{K} \sum_{l=1}^{L} \beta_{k_{l}}^{q} x_{w_{n i t k_{l}}}\right)\right]}{\sum_{j=1}^{J} \exp \left[\left(\sum_{k=1}^{K} \sum_{l=1}^{L} \beta_{k_{l}}^{q} x_{b_{n j t k_{l}}}\right)-\left(\sum_{k=1}^{K} \sum_{l=1}^{L} \beta_{k_{l}}^{q} x_{w_{n j k_{l}}}\right)\right]} .
$$

Given the strong evidence that a significant proportion of respondents ignore one or more of the attributes (e.g., Hensher et al., 2005; Campbell et al., 2008), it is, therefore, appropriate to also accommodate rationally adaptive behaviour in the form of different attribute processing strategies. For this reason, we impose the condition that $\beta_{k}^{M_{k}}$ is a zero vector, where the parameters for all $L$ attribute levels for attribute $k$ are fixed to zero.

Owing to the risk of confounding between preferences and non-attendance (e.g., see Campbell et al., 2012; Hensher et al., 2012a; Hess et al., 2012, for an overview of the issues) it is important to simultaneously address preference heterogeneity. Although this is unlikely to resolve this confounding issue, it should certainly reduce it. This is because allowing for preference heterogeneity increases the probability that the classes with a zero coefficient is a true reflection of non-attendance, and not a result of confounding non-attendance with 'weak' preferences. Having said that, it is important to recognise the difficulties in separating non-attendance and indifferences in preferences (i.e. one cannot distinguish between the case where a zero coefficient is the outcome of a simplifying heuristic and where it is instead a true manifestation of individual preferences). Taking this into account, and after evaluating the results from various specifications and distributional assumptions, for each attribute we assume three support points (two of which are estimated and one that is fixed at zero), leading to $Q=3 \times 3 \times 3=27$ possible segments. Although, in this paper, we assume discrete distributions, we recognise the work by Campbell and Doherty (2013) where discrete and continuous distributions are accommodated concurrently.

Models are estimated using maximum likelihood estimation and in the case of the discrete mixtures model that retrieves class probabilities, we are mindful of its vulnerability to local maxima of the sample-likelihood function. Thus, in an attempt to reduce the possibility of reaching a local, rather than a global, maximum, we started the estimation iterations from a variety of random starting points. Specifically, we do this by estimating the model many times (at least 1,000 times), but each time using a different vector of starting values, which is chosen randomly.

\footnotetext{
${ }^{7}$ We note that student-specific characteristics can be included as covariates to help establish profiles of students who are associated with each mass point by specifying $\pi_{k}^{m}$ as: $\pi_{k}^{m}=\exp \left(c_{k}^{m}+\psi_{k}^{m} z_{n}\right) / \sum_{m=1}^{M_{k}} \exp \left(c_{k}^{m}+\psi_{k}^{m} z_{n}\right)$, where $c_{k}^{m}$ is a constant for support point $m$ for attribute $k$ and $\psi_{k}^{m}$ are parameters relating to the student's characteristic $z$. As part of our initial estimations we included several covariates. However, none came out as significant, which is expected given the relatively small sample size and, relatedly, small sample variation in the covariates. Additionally, we also found that adding including these extra parameters led to a proliferation of parameters and, therefore, loss of parsimony, which we considered to be a paramount concern given our final sample size.
} 


\section{Results}

Table 2 reports estimation results obtained from two model specifications. The first model relates to the multinomial logit model (Eq. 2), whereas the second model is the discrete mixtures model (Eq. 3).

Looking firstly at the multinomial logit model results, we find that, compared to modules delivered in ten 4-hour sessions, students, on average, dislike modules delivered via forty 1-hour sessions but, all else held constant, significantly prefer modules that are delivered in twenty 2-hour sessions. Indeed, on the basis of the marginal utility parameters for the delivery attribute levels, we can say that, if offered the choice between three modules that differed only in terms of delivery, almost 60 percent of students would choose the module delivered in twenty 2-hour sessions. Prima facie, our results also suggest that students appear to prefer modules that involve tutorials and are averse to those that entail lab sessions. We remark though, that the marginal utilities are not significantly different from each other, meaning that, statistically speaking, student's preferences for the three teaching attribute levels are not dissimilar. In accordance with Donaldson and McNicholas (2004), we find the method of assessment to be an important consideration in the students' choices. On average, students have a clear (and statistically significant) preference for modules that do not have examinations that contribute 100 percent towards the overall module grade. As a matter of fact, using the marginal utility parameter estimated for the assessment attribute, if offered a choice between three modules where all other factors are held constant, less than one-fifth of students would choose the module where there is no coursework assessment. In contrast, almost half of the students would choose the module where the coursework assessment accounts for 40 percent of the overall module grade.

While the estimates retrieved under the multinomial logit model give an important insight into student's preferences for different module designs, this is based on the premise that all students share the same preferences. For a variety of reasons, one could postulate the hypothesis of preference heterogeneity and the presence of attribute processing strategies. For this reason, we now turn our attention to the results for the discrete mixtures model. We find this leads to a large increase in model fit (by almost 30 log-likelihood units). Moreover, although estimating additional support points and the probabilities associated with these support points comes at a very high parametric cost, the $\bar{\rho}^{2}$, as well as the Akaike information criterion, confirm

Table 2

Estimation results

\begin{tabular}{|c|c|c|c|c|}
\hline & Multinomial logit & & Discrete mixtures & \\
\hline & & $m=1$ & $m=2$ & $m=3$ \\
\hline & est. (std. err.) & est. (std. err.) & est. (std. err.) & est. (std. err.) \\
\hline Delivery & & & & \\
\hline Forty 1-hour sessions & $-0.440^{* * *}(0.139)$ & $-1.164^{* *}(0.558)$ & $-1.178^{* * *}(0.391)$ & 0.000 \\
\hline Twenty 2-hour sessions & $0.728^{* * *}(0.154)$ & $1.984^{* * *}(0.617)$ & $-1.229^{* * *}(0.461)$ & 0.000 \\
\hline Ten 4-hour sessions & $-0.288^{* *}(0.119)$ & $-0.820^{* *} \quad(0.351)$ & $2.406^{* * *}(0.685)$ & 0.000 \\
\hline Probability share & 1.000 & $0.409^{* *}(0.178)$ & $0.176^{* * *}(0.071)$ & $0.415^{* * *}(0.162)$ \\
\hline Teaching & & & & \\
\hline Lectures and lab sessions & $(0.074)$ & $(0.170)$ & $(0.213)$ & 0.000 \\
\hline Lectures and tutorials & $(0.126)$ & $(0.162)$ & $(1.810)$ & 0.000 \\
\hline Lectures, lab sessions and tutorials & $(0.105)$ & $0.455^{* * *}(0.175)$ & $(1.697)$ & 0.000 \\
\hline Probability share & 1.000 & $0.799^{* * *}(0.180)$ & $(0.083)$ & $(0.167)$ \\
\hline Assessment & & & & \\
\hline $100 \%$ exam and $0 \%$ coursework & $-0.508^{* * *}(0.111)$ & $-1.443^{* * *}(0.464)$ & $-1.994^{* *}(0.892)$ & 0.000 \\
\hline $80 \%$ exam and $20 \%$ coursework & $-0.127 \quad(0.135)$ & $0.157 \quad(0.543)$ & $(1.602)$ & 0.000 \\
\hline $60 \%$ exam and $40 \%$ coursework & $0.381^{* * *}(0.122)$ & $1.286^{* * *}(0.443)$ & $(0.739)$ & 0.000 \\
\hline Probability share & 1.000 & $0.366^{* *}(0.166)$ & $0.293^{* *}(0.165)$ & $0.341^{* * *}(0.098)$ \\
\hline Log-likelihood & -464.801 & & -435.360 & \\
\hline $\mathrm{K}$ & 6 & & 18 & \\
\hline $\bar{\rho}^{2}$ & 0.443 & & 0.464 & \\
\hline Akaike information criterion & 941.602 & & 906.719 & \\
\hline
\end{tabular}

Note: All estimated standard errors are robust and clustered at the student level. ${ }^{*},{ }^{* *}$ and ${ }^{* * *}$ indicate statistical significance at the 10,5 and 1 percent level respectively. 
this improvement in model performance even after accounting for the loss of parsimony. We acknowledge that this improvement is, in part, due to the fact that the panel nature of the data is being accounted for, it is difficult to truly corroborate this.

Scrutiny of the segment-specific marginal utility parameters retrieved under the discrete mixtures model provides a clear signal that not all students share the same preferences for the module attributes and that a sizeable proportion ignored the course attributes. Among students who are predicted to have considered the delivery attribute, the majority ( 41 percent of all students) are found to have a very strong preference modules delivered in 2-hour blocks. In fact, if offered a choice between modules that differed solely on the basis of how it is delivered, the estimated coefficients imply that over 90 percent of these students would choose to enrol in the course delivered in 2-hour sessions. Interestingly, an entirely different implied ranking of the levels is observed for the students associated with the second set of mass points estimated for the delivery attribute (which accounts for 18 percent of students). Only 3 percent of these students would chose the module delivered in 2-hour blocks. The overwhelming majority (95 percent) of these students would register in the course delivered in longer blocks of 4-hours. Findings obtained from the multinomial logit model give the impression that the teaching attribute is not an important feature of module preference and is not influencing choice. The more flexible discrete mixtures model does not support this. Approximately 80 percent of students share the same marginal utilities for the teaching attribute levels, indicating a lower degree of preference heterogeneity. Within this segment, there is a clear preference for modules that are a combination of lectures, computer lab sessions and tutorials: approximately half of students in this segment would choose to enrol in such a module. The second segment have distinctly different preferences. In fact, they appear to have a very strong dislike of modules that include computer lab sessions, but instead prefer modules that only offer tutorials. It is acknowledged that the differences in marginal utilities for the three levels of the teaching attribute within this segment are not significant, which is not surprising given that this is a relatively small segment (comprising of fewer than 10 percent of all students). Notable differences are observed between the two segments who are predicted as having attended to the assessment attribute. While both segments have a significant marginal dis-utility for modules where the examination contributes 100 percent towards the overall grade, the first segment (consisting of 37 percent of students) prefer a larger coursework assessment component compared to the second segment (29 percent of students): respectively, 86 percent and 23 percent of students in the first and second segments would choose modules with the larger coursework assessment component. We also draw attention to the existence of attribute non-attendance among students - the unconditional probabilities suggest that 42 percent, 10 percent (albeit not significant) and 34 percent of students ignored the delivery, teaching and assessment attributes, respectively. For these students, the respective attribute has no influence on the module they choose to enrol in.

To further tease out what these results mean for students' choice of modules, we explore choice probabilities for a number of specific module profiles using the results from both models. This analysis uses the estimated marginal utility parameters and, in the case of the discrete mixtures model, the unconditional segment membership probabilities. For this analysis, in order to clearly demonstrate the differences between segments, we consider the three module profiles portrayed in Figure 1. Expected values and 95 percent confidence intervals from this post-estimation analysis (based on 100,000 simulated draws) are given in Figure 2.

Looking firstly at the predicted shares for module 1 in Figure 2(a), we find an apparent difference between the shares predicted using the coefficients retrieved from the multinomial logit and discrete mixtures models, thus demonstrating the implications of the unrealistically simple assumptions of the inferior model. Specifically, the multinomial logit model overpredicts compared to the discrete mixtures model (respectively, the models predict 40 and 34 percent will choose to enrol in this module). The overall prediction from the discrete mixtures is a weighted average (using the unconditional segment probabilities) over the 27 possible segments. This misses opportunities for insight since it masks the striking differences we observe between different mass points. The predictions attained for students conditional on the three mass points associated with the delivery attribute are, statistically different. Students whose preferences for the delivery attribute are represented by the first mass point are highly likely to choose this module-indeed, based on the expected value, almost two-thirds of these students will choose it - which is not surprising given their strong preference for modules delivered in twenty 2-hour sessions. In contrast, less than 3 percent of students whose preferences 


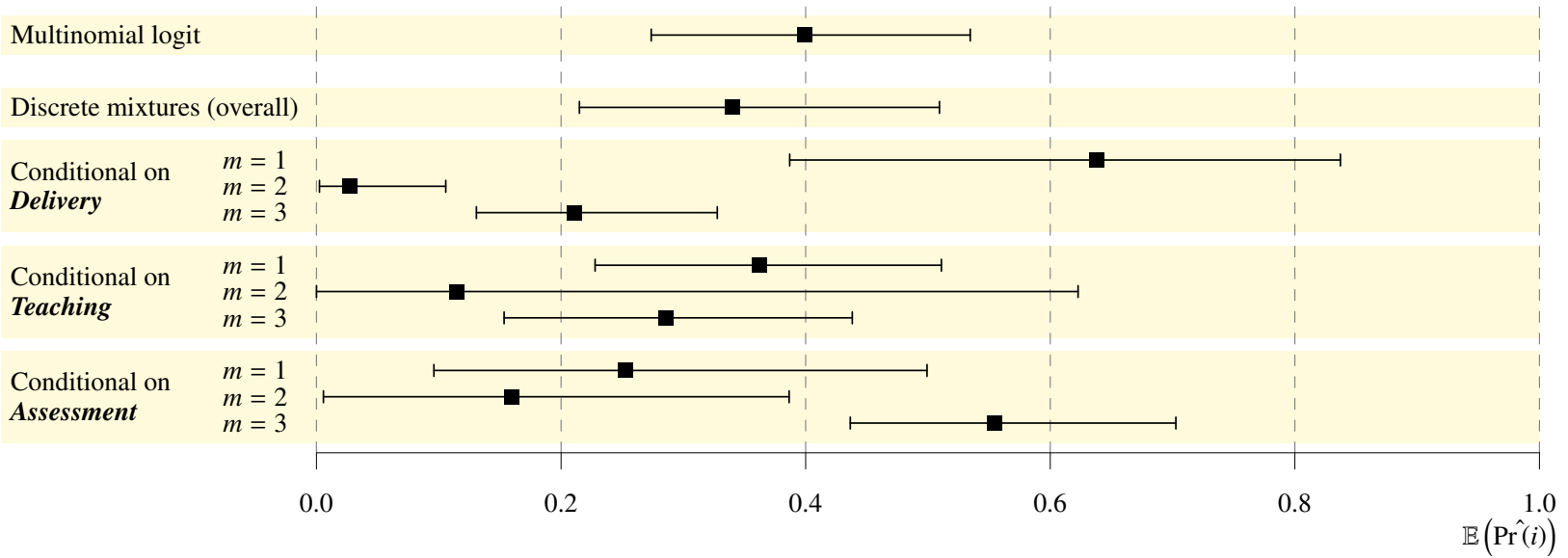

(a) Module 1 (twenty 2-hour sessions; lectures, lab sessions and tutorials; and, 100\% exam and 0\% coursework)

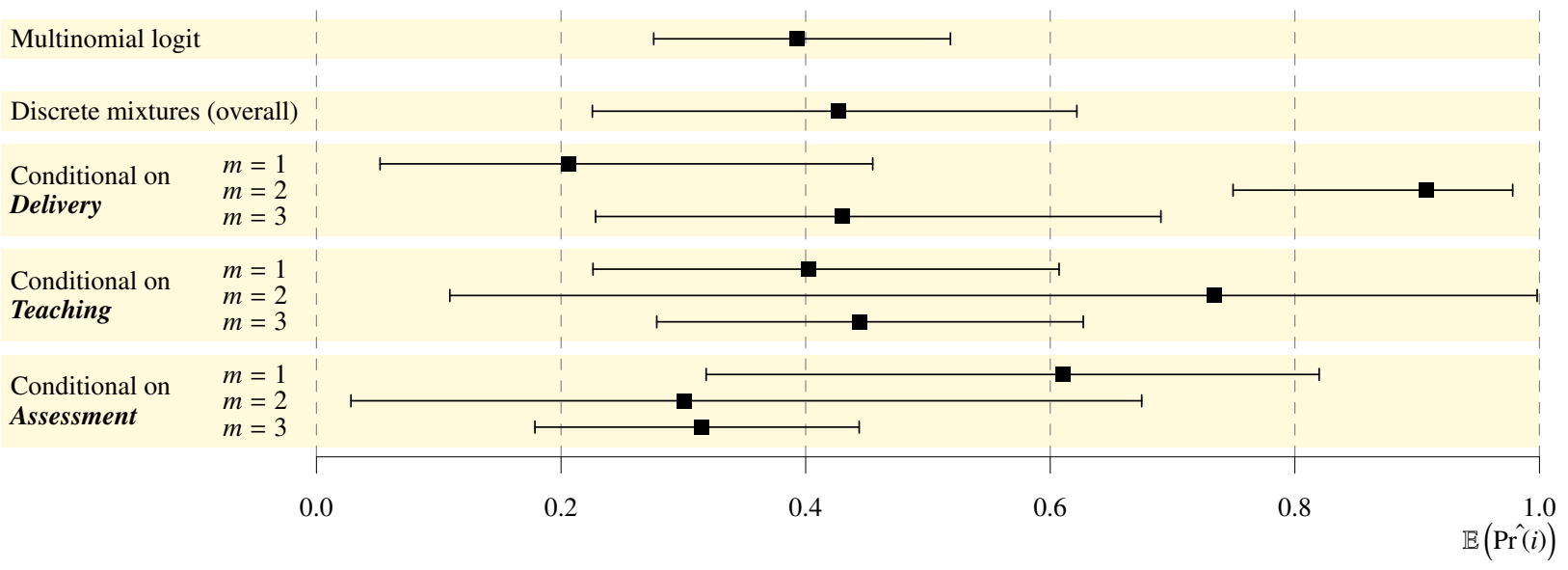

(b) Module 2 (ten 4-hour sessions; lectures and tutorials; and, 60\% exam and 40\% coursework)

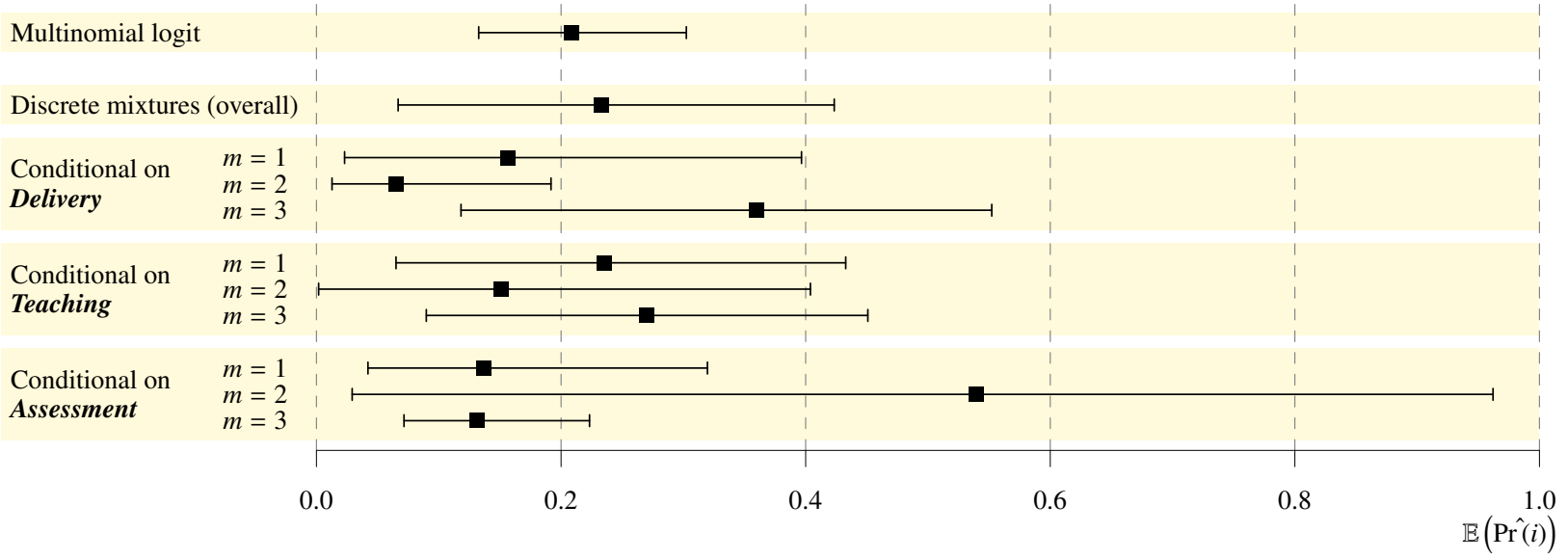

(c) Module 3 (forty 1-hour sessions; lectures and lab sessions; and, $80 \%$ exam and 20\% coursework)

Figure 2: Scenario probabilities (expected values with error bars showing the 95 percent confidence interval based on 100,000 simulated draws)

for this attribute are given by the second mass point are predicted to enrol in this module. Among students who ignore the delivery attribute, the expected value implies that just over one-fifth are likely to choose module 1. The differences in predicted choice shares for this module attained for the three mass points for teaching are not statistically different from each other. Nevertheless, it is interesting to note that 36 percent of students who belong in the first segment (i.e., those who prefer a combination of lectures, computer lab sessions and tutorials) are predicted to enrol in this module, compared to 11 and 28 percent of students 
whose preferences are given by the second and third segment correspondingly. Interestingly, given that the assessment for module 1 is based on 100 percent examination and most students exhibited dis-utility for this marking structure, only students who ignore the assessment attribute are likely to select it (the predicted share is 55 percent). This goes some way to explaining why the multinomial logit model (which does not account for attribute non-attendance) overestimates the share of students who will choose this module. We find that students whose preferences for assessment attribute are given by the first and second mass points are respectively, 25 and 16 percent likely to choose module 1 as their most preferred.

Comparing the probability shares estimated for module 2, presented in Figure 2(b), we, again, observe a difference between the multinomial logit and discrete mixtures model. In this case, however, we find that the multinomial logit model (39 percent) underpredicts compared to the overall discrete mixtures model (43 percent). While this divergence may not be as large compared to that observed for module 1, it has important implications. Remark that, on average, the naïve multinomial logit model suggests that students are slightly more likely to choose module 1 compared to module 2 , but under the more insightful discrete mixtures model this is reversed. Under this model, students are on average, found to have a clear preference (in the magnitude of almost 10 percent) for module 2. This corroborates our decision to recognise the heterogeneous nature of students' preferences and their possible lexicographic preference orderings. Assessing the probabilities for module 2 that are conditional on the three mass points obtained for the delivery attribute, we observe a significantly higher prediction (over 90 percent) for students who have a positive marginal utility for modules delivered in ten 4-hour sessions (i.e., the second mass point). Students whose preferences for the delivery attribute are given by the first mass points are least likely to choose this module (just over 20 percent). Based on the expected value, 43 percent of students who ignore the delivery attribute will choose this module. Students belonging in the first teaching segment have relatively weak preferences for the lecture and tutorial levels, so it is not unexpected to find that the majority of these students are unlikely to choose module 2 (i.e., nearly 60 percent would not select this module). In contrast, the second segment find this module very appealing (with almost three-quarters of this subset predicted to enrol in this module). This said, it is recognised that the confidence interval is quite wide, which is an artefact of the relatively small proportion of students who hold these preferences. Conditional on ignoring the teaching attribute, 44 percent of students are likely to choose this module. Students whose preferences for the assessment attribute are denoted by the first mass point are highly likely to choose this module, which reflects the relatively high weight these students place on this grading structure when making their choices. The predicted shares of students who would choose module 2 are found to be relatively similar (30 and 31 percent, respectively) for the second and third assessment segments.

Finally, while the predicted shares for module 3 can be deduced (since the probability share for the three modules sum to one), we, nevertheless, present Figure 2(c) to ease interpretation. On average, this is found to be the least popular module, with less than one-quarter of students likely to enrol in it. Once more, the multinomial logit model underpredicts relatives to the discrete mixtures model (respectively, 21 percent vis-à-vis 23 percent). Results presented in Table 2 showed that students in the first two delivery segments find modules delivered via forty 1-hour session relatively unappealing. Students in both segments are, therefore, found to be unlikely to choose module 3 . This module is much more likely to be chosen by students who ignore the delivery attribute. We remark that a similar pattern is produced using the three mass points retrieved for the teaching attribute, albeit with less remarkable differences across segments since all three predicted probabilities are less than 30 percent. All else held constant, students whose preferences are given by the second assessment segment have a clear preference for modules where their overall grade is weighted according to a 80:20 examination to coursework ratio, meaning that these students are the most likely to choose module 3 . The probabilities for the remaining two assessment segments are comparable (in the region of 13-14 percent).

\section{Conclusions}

In this study, we estimate postgraduate economics students' preferences for module design through administering and analysing a multi-profile best-worst scaling survey. We find that students have clear preferences on how they wish their modules to be delivered. They dislike block teaching and, similarly, very short sessions, 
but instead prefer something in-between. We also reveal that postgraduate economics students prefer tutorials over lab sessions and that they find modules that are assessed only by a final examination as unappealing compared to modules that involve an element of coursework assessment. Furthermore, we find heterogeneous preferences for module attributes among students.

Although based on 944 students' choices, we note the relatively small sample is a limitation of the study. While this does not preclude us from estimating the student choices and reaching our conclusions, we recognise that a larger sample of students would have given even greater credence to our findings. This said, it is clear from this sample that there is a significant degree of heterogeneity among students and a sizeable share of students who ignore certain aspects of modules when choosing their elective modules. We show that failing to account for this heterogeneity in students' preferences and these lexicographic preference orderings results in erroneous results and, crucially, limits the ability to estimate reliable predictions of enrolment in different modules. University staff responsible for predicting the demand for different modules should be especially wary of this phenomenon when planning course design. Although it may not be realistic to always take student preferences into account when designing course modules, small efforts can be made to improve student satisfaction.

Undoubtedly, the findings in this study should appeal to university staff involved in the design of postgraduate (as well as undergraduate) courses. The results here clearly show that student satisfaction depends not only on module content, but also on the method of delivery, teaching, and assessment. Importantly, in terms of the many national surveys used as a barometer of student satisfaction (such as the National Student Survey and Postgraduate Taught Experience Survey conducted in the UK) and module evaluations, students will be more likely to provide positive feedback and score the modules more favourably if modules are designed with their preferences in mind. Obviously, it is not always feasible (nor appropriate) to tailor modules according to students' preferences. Modules must fit within the overall programme and their curricula needs to be shaped so that the teaching methods and assessment tasks align with the intended learning outcomes. Modules must also provide a coherent learning experience for students and facilitate the achievement of the intended learning outcomes associated with them. Several studies have examined the link between learning outcomes and teaching styles and methods of delivery. While this goes beyond the scope of this paper, it is worthwhile to note that different student learning styles can affect student achievement. Indeed, Lage et al. (2000) find that the divergence between teaching and learning styles can result in students learning less or becoming disinterested in the subject. Moreover, many studies (e.g., Duque, 2014; Strahan and Credé, 2015) report a relationship not just between student satisfaction and affective learning outcomes, but also with dropout intentions. Therefore, a further extension of this research would be to examine the link between module preferences and student learning outcomes and dropout intentions. Notwithstanding this, this study highlights the module characteristics that are widely less appealing from the students' perspective and, therefore, are best avoided or minimised when possible. Furthermore, this study shows that module design should vary in order to increase the likelihood that all students' preferences are met. Armed with this information, universities should be better positioned to deliver efficient course structures, tailored to the learning styles of economic students, through which students can attain their full academic potential.

\section{References}

Alemu, M., Mørkbak, M., Olsen, S. and Jensen, C. (2013). Attending to the reasons for attribute nonattendance in choice experiments, Environmental and Resource Economics 54(3): 333-359.

Campbell, D. and Doherty, E. (2013). Combining discrete and continuous mixing distributions to identify niche markets for food, European Review of Agricultural Economics 40(2): 287-311.

Campbell, D., Hensher, D. and Scarpa, R. (2011). Non-attendance to attributes in environmental choice analysis: a latent class specification, Journal of Environmental Planning and Management 54(8): 10611076.

Campbell, D., Hensher, D. and Scarpa, R. (2012). Cost thresholds, cut-offs and sensitivities in stated choice analysis: Identification and implications, Resource and Energy Economics 34(3): 396-411.

Campbell, D., Hutchinson, W. and Scarpa, R. (2008). Incorporating discontinuous preferences into the analysis of discrete choice experiments, Environmental and Resource Economics 41(3): 401-417. 
Canning, J. (2015). A new measurement and ranking system for the UK national student survey, Perspectives: Policy and Practice in Higher Education 19(2): 56-65.

Donaldson, B. and McNicholas, C. (2004). Understanding the postgraduate education market for UK-based students: a review and empirical study, International Journal of Nonprofit and Voluntary Sector Marketing 9(4): 346-360.

Douglas, J. A., Douglas, A., McClelland, R. J. and Davies, J. (2015). Understanding student satisfaction and dissatisfaction: an interpretive study in the UK higher education context, Studies in Higher Education 40(2): 329-349.

Dunnett, A., Moorhouse, J., Walsh, C. and Barry, C. (2012). Choosing a university: a conjoint analysis of the impact of higher fees on students applying for university in 2012, Tertiary Education and Management 18(3): 199-220.

Dunnett, A., Moorhouse, J., Walsh, C. and Barry, C. (2014). A framework for analysing higher education performance: students' satisfaction, perceived learning outcomes, and dropout intentions, Total Quality Management and Business Excellence 25(1): 1-21.

Finn, A. and Louviere, J. J. (1992). Determining the appropriate response to evidence of public concern: the case of food safety, Journal of Public Policy and Marketing 11(2): 12-25.

Flannery, D., Kennelly, B., Doherty, E., Hynes, S. and Considine, J. (2013). Of mice and pens: a discrete choice experiment on student preferences for assignment systems in economics, International Review of Economics Education 14: 57-70.

Hagel, P. and Shaw, R. N. (2010). How important is study mode in student university choice?, Higher Education Quarterly 64(2): 161-182.

Hensher, D. A., Collins, A. T. and Greene, W. H. (2012a). Accounting for attribute non-attendance and common-metric aggregation in a probabilistic decision process mixed multinomial logit model: a warning on potential confounding, Transportation 40(5): 1003-1020.

Hensher, D., Rose, J. and Greene, W. (2005). The implications on willingness to pay of respondents ignoring specific attributes, Transportation 32: 203-222.

Hensher, D., Rose, J. and Greene, W. (2012b). Inferring attribute non-attendance from stated choice data: implications for willingness to pay estimates and a warning for stated choice experiment design, Transportation 39(2): 235-245.

Hess, S., Bierlaire, M. and Polak, J. W. (2007). A systematic comparison of continuous and discrete mixture models, European Transport 37: 35-61.

Hess, S. and Hensher, D. (2013). Making use of respondent reported processing information to understand attribute importance: a latent variable scaling approach, Transportation 40(2): 397-412.

Hess, S., Stathopoulos, A., Campbell, D., O’Neill, V. and Caussade, S. (2012). It's not that I don't care, I just don't care very much: confounding between attribute non-attendance and taste heterogeneity, Transportation 40(3): 583-607.

Ho, A., Kember, D. and Hong, C. (2012). What motivates an ever increasing number of students to enroll in part-time taught postgraduate awards?, Studies in Continuing Education 34(3): 319-338.

Holdsworth, D. K. and Nind, D. (2006). Choice modeling New Zealand high school seniors' preferences for university education, Journal of Marketing for Higher Education 15(2): 81-102.

Kember, D., Ho, A. and Leung, D. Y. (2016). Evaluating taught postgraduate awards from the student's perspective, Journal of Further and Higher Education 40(2): 147-169.

Lage, M. J., Platt, G. J. and Treglia, M. (2000). Inverting the classroom: a gateway to creating an inclusive learning environment, Journal of Economic Education 31(1): 30-43.

Lancaster, K. J. (1966). A new approach to consumer theory, Journal of Political Economy 74(2): 132-157.

Leman, J., Turner, G. and Bennett, P. (2013). Ptes 2013: Findings from the postgraduate taught experience survey, Higher Education Academy, York.

Lemieux, T. (2006). Postsecondary education and increasing wage inequality, American Economic Review 96(2): 195-199.

Louviere, J. J., Flynn, T. N. and Marley, A. A. J. (2015). Best-worst scaling: theory, methods and applications, Cambridge University Press, Cambridge.

Manski, C. F. (1977). The structure of random utility models, Theory and Decision 8: 229-254. 
McFadden, D. (1974). Conditional logit analysis of qualitative choice behavior, in P. Zarembka (ed.), Frontiers in Economics, Academic Press, New York.

McFadden, D. (2001). Economic choices, American Economic Review 91(3): 351-378.

Morgan, M. (2014). Patterns, drivers and challenges pertaining to postgraduate taught study: an international comparative analysis, Higher Education Research E Development 33(6): 1150-1165.

Scarpa, R., Gilbride, T., Campbell, D. and Hensher, D. (2009). Modelling attribute non-attendance in choice experiments for rural landscape valuation, European Review of Agricultural Economics 36(2): 151-174.

Scarpa, R., Zanoli, R., Bruschi, V. and Naspetti, S. (2013). Inferred and stated attribute non-attendance in food choice experiments, American Journal of Agricultural Economics 95(1): 165-180.

Strahan, S. and Credé, M. (2015). Satisfaction with college re-examining its structure and its relationships with the intent to remain in college and academic performance, Journal of College Student Retention: Research, Theory and Practice 16(4): 537-561.

Thurstone, L. L. (1927). A law of comparative judgment, Psychological Review 34: 273-286. 